\title{
PHOTOACOUSTIC DETECTION OF CIRCULAR DICHROISM IN A SQUARE ARRAY OF NANO-HELICES
}

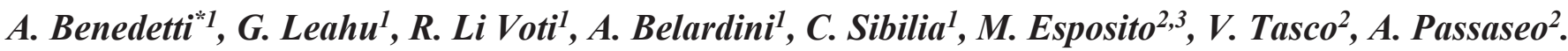 \\ ${ }^{1}$ Sapienza Università di Roma, Department S.B.A.I. Physics Section, Via A. Scarpa 14, I-00161 Roma, Italy \\ ${ }^{2}$ National Nanotechnology Laboratory, CNR Nanotech, Via Arnesano, I-73100 Lecce, Italy. \\ ${ }^{3}$ Università del Salento, Via Arnesano, I-73100 Lecce, Italy. \\ * corresponding author: alessio.benedetti@uniroma1.it
}

\begin{abstract}
A novel nano-structured material has been assembled by means of a focused ion beam technique. This artificial material is composed of a square array of nano-helices built upon a multilayered substrate. Optical measurements of circular dichroism of a sample are confirmed by photo-acoustic investigations, which allow to directly study the helix-field interaction apart from the dielectric substrate. The study is consistent with 3D numerical simulations, and demonstrates to be an efficient tool of investigation for the entire class of these novel structured materials.
\end{abstract}

\section{Introduction.}

Circular Dichroism (CD) relies on the differential absorption of left and right circularly polarized waves. Sometimes it is more generally referred to as the different interaction of a sample with waves featuring the two opposite senses of rotation. This phenomenon occurs naturally with materials possessing intrinsic chirality [1], and since the most prominent chiral geometry is the one of the helix, we focused our efforts for the construction of a nano-structured material composed of many helices disposed along a square grid in order to promote CD at optical frequencies $[2,3,4]$.

The method used to build these kind of artificial materials is basically consisting of a focused ion beam technique [2,3], and takes advantage of the high precision and energy of the ion beam to correctly design complicated structures. The final result is an ordered collection of helices made of a platinum-carbon $(\mathrm{Pt}-\mathrm{C})$ mixture grown upon a dielectric substrate. A continuous feedback between numerical simulations and experimental tests allowed to choose the correct set of parameters for the construction of a class of samples showing strong $\mathrm{CD}$ values at optical frequencies. The technology used is very scalable, and further samples can be constructed to be functional also in the UV ranges.

The CD in this sample have been already measured $[3,4]$, but the all-optical measurements suffer from the small spatial extension of the samples in comparison with the totally illuminated region, since the transmission spectra are averaged with the transmitted field emerging from an illuminated portion of pure substrate. Furthermore, in order to calculate the absorption spectra, even the reflectivity must be measured. The photoacoustic (PA) technique allows to overcome these problems by taking advantage of the sound promotion by ohmic losses, which occur only in the helices' region. Despite the sound signal being weak for the low excitation process, the different registered sound levels are consistent with the different levels of absorption for the two opposite senses of circular polarization.
Full simulations and a 3D map of the ohmic losses across the surface allow to better comprehend the field-toheat generation and why consistent differences of sound levels appear when illuminating the sample either with an upward or downward oriented beam. These numerical estimates are consistent with the PA measurements, which show to be an efficient way to better evaluate the absorption process for this kind of artificial materials and complementary to all-optical measurements.

In section 2 of this paper, we briefly discuss about the PA technique, and we show the generic adopted scheme for the PA measurements. In section 3, we present our numerical studies, while in section 4 we plot the main experimental data, and a comparison with the theoretical results is provided. Finally, in section 5 the main conclusions are drawn.

\section{Photoacoustic Technique and Apparatus.}

The concept of converting the power consumed by ohmic losses into sound waves is not a novel idea, but rather it was implemented by some authors many years ago $[5,6]$ to study the absorption spectra of some lossy media. Anyway, the advent of affordable instruments for nanotechnology [7] allowed to produce structured samples in the nano-scale only recently, and the use of the $\mathrm{PA}$ technique to reveal $\mathrm{CD}$ in chiral media has become incredibly more appealing.

The complex mechanism of the e.m.-to-sound conversion has been intensively studied, and it proceeds following many paths [6]. Essentially it consists in the transmission of heat from the sample' hot regions across the surface to the air by direct contact. The heat transfusion to the air background can proceed by other means, for instance by direct radiative emission, but this latter phenomenon is less efficient (see fig.1) [6]. 


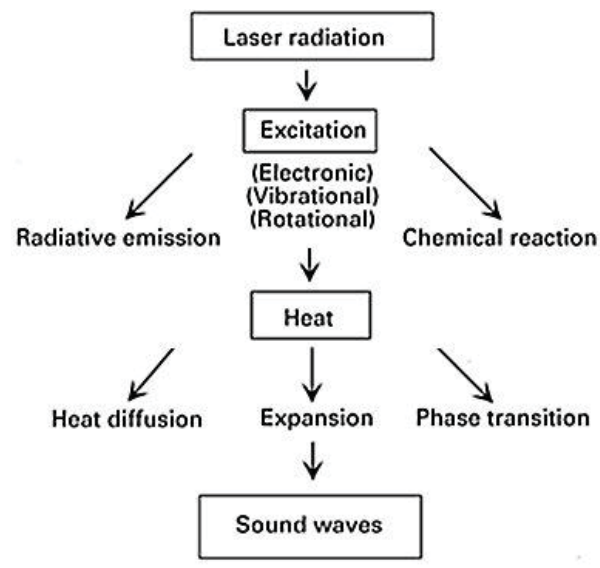

Figure 1. Schematic representation of the different processes leading to the PA signal generation. The absorbed e.m. field is partially converted into heat and further partially transformed into acoutic signal.

The periodic increase and decrease of the air differential volume in dependence of the major/minor e.m. dissipation gives rise to periodic air oscillations which propagates at the sound speed, and which can be converted into a recordable electric signal by a sensitive microphone located into the PA cell. In order to make the sound perturbation audible by the microphone, the e.m. wave must be modulated at suitable frequencies. We thus added a chopper to the experimental setup and modulated our input e.m. laser source at $25 \mathrm{~Hz}$, and measured the sound produced at that frequency.

A schematics view of the entire experimental set-up is shown in fig.2. Among the other parts, it is clearly observable the laser source, the chopper, the polarizing lens, and the PA cell.
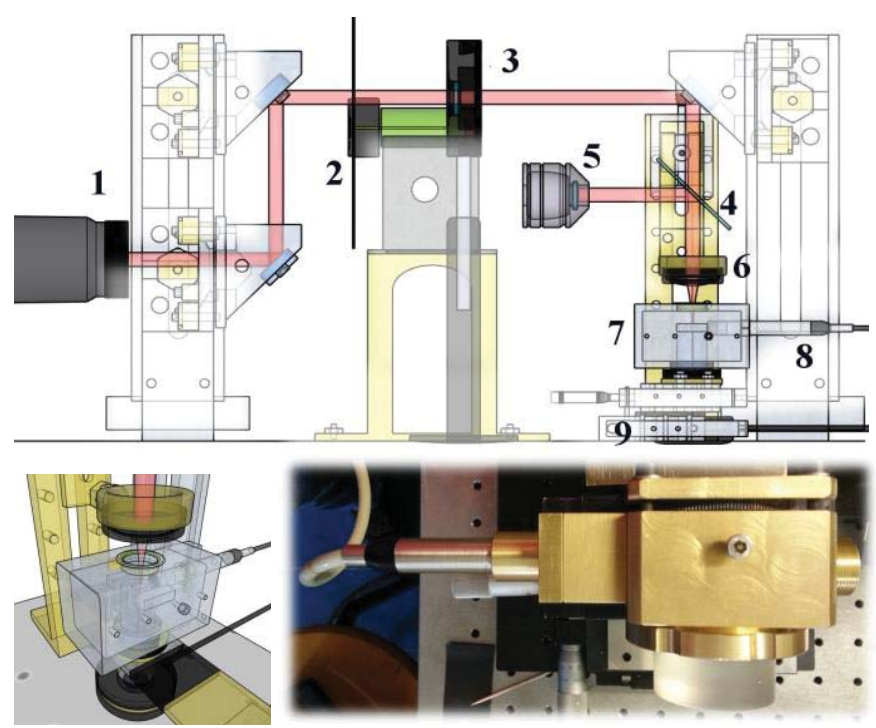

Figure 2. In the top, schematic view of the PA set-up: 1-laser source, 2 chopper, 3-polarizing lens, 4-beam splitter, 5-microscope objective, 6 focusing lens, 7-PA cell, 8-microphone, 9-photodetector. A magnified visual of the PA cell is presented (bottom left), with an actual pitcure of the same (bottom right) showing also the Brüel \& Kjær acoustic set at its left side.

After many passages, the laser crosses a chopper (which modulates the laser source at $25 \mathrm{~Hz}$ ) and a polarizing lens in order to promote circular polarization. A BS and a microscope allow to properly view and consequently point the laser toward the sample's exact location inside the PA cell. This latter contains also a sensitive microphone (a Brüel \& Kjær ANSI Type-4166 [8] with a Brüel \& Kjær PreAmplifier Type-2619) to detect the photoacoustic generated sound wave at $25 \mathrm{~Hz}$. Finally, a photodetector located at the end of the laser line allows further optical measurements in transmission.

\section{Numerical data.}

The helices are made of a Pt-C mixture due to the particular construction process. The percentages are approximatively $20 \%$ of Platinum and $80 \%$ of Carbon, although the precise composition of the compounding matter is unknown. Anyway, we already demonstrated that the spectral properties of this artificial material are prevalently dominated by the helix shape rather than its chemical composition [3,4], so small variation of the materials' percentages are quite negligible and allowed when trying to evaluate the main spectra with sufficient precision.

The helices are composed by a 3-turned $130 \mathrm{~nm}$ diameter filament, and they feature $95 \mathrm{~nm}$ skeleton radius and $330 \mathrm{~nm}$ vertical pitch (see fig.3a). The helices are located at the nodes of a square lattice with a period of $700 \mathrm{~nm}$ (see fig. $3 \mathrm{~b}$ ). The construction process has been performed upon a multipurpose substrate, which is composed by a superposition of 3 layers made of $\mathrm{Al}_{0.3} \mathrm{Ga}_{0.7} \mathrm{~N}, \mathrm{GaN}$ and $\mathrm{AlN}$, with thickness values of $20 \mathrm{~nm}, 2 \mu \mathrm{m}$, and $100 \mathrm{~nm}$, respectively, upon a final substrate of $\mathrm{Al}_{2} \mathrm{O}_{3}$, with a thickness of several mm.

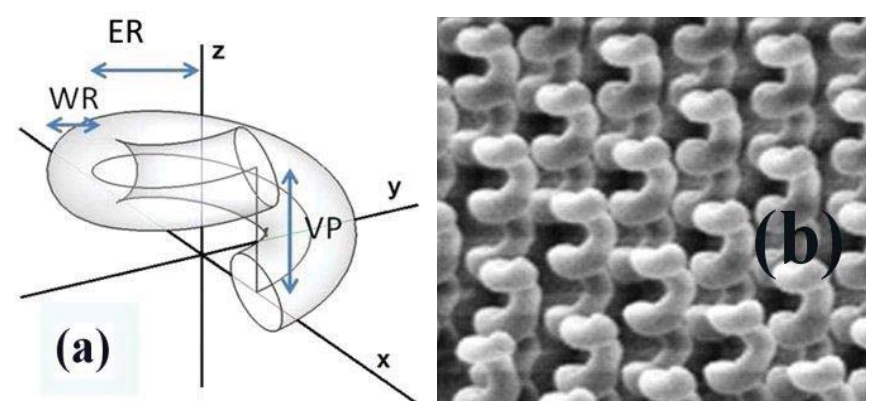

Figure 3. (a) Geometric representation of the helix with its main geometric parameters. $\mathrm{WR}=65 \mathrm{~nm}, \mathrm{ER}=95$, and $\mathrm{VP}=330 \mathrm{~nm}$. (b) $\mathrm{SEM}$ image of the sample. It is clearly visible the triple turn for each single helix. The suqare lattice has a periodic step of $700 \mathrm{~nm}$.

In order to have a complete set of numerical data, we both estimated the far-field transmission $(\mathrm{T})$ and reflection (R) spectra, together with the total absorption (A) spectrum. The former spectra have been evaluated extrapolating the fundamental component (00-mode) from the field distribution, thus deleting any Bragg contribution for all the wavelengths below 700nm, while the latter has been calculated subtracting the total reflectance and transmittance at the unitary value. These spectra are shown in fig.4, with the linear polarization spectra also included. 


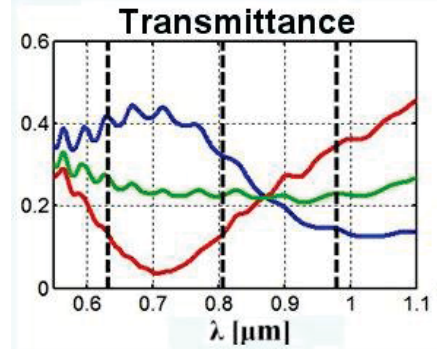

(a)

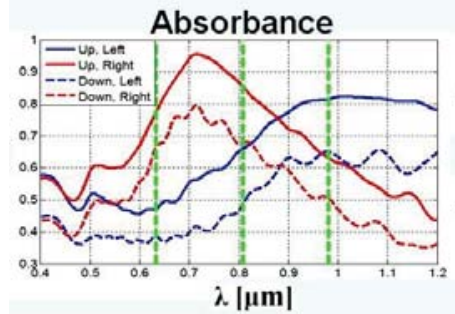

(c)

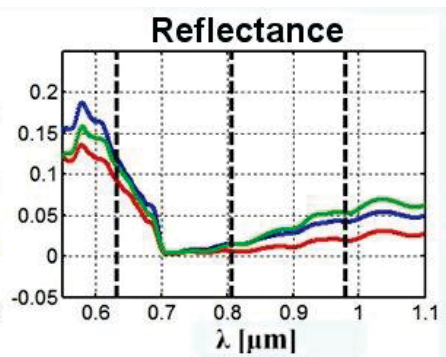

(b)

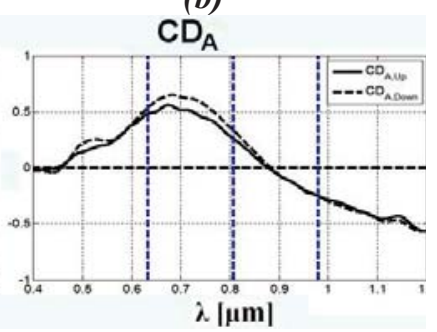

(d)
Figure 4. Transmission (a) and reflection (b) spectra of the fundamental field component. Red, green and blue lines refer to the right circular, linear, and left circular polarizations, respectively. These spectra have been calculated by a virtual incidence from above the helices' layer. The corresponding absorption spectra is plotted in (c) with straight lines. In the same picture we also plotted the absorption spectra when the sample is illuminated from below the substrate. (d) $\mathrm{CD}_{\mathrm{A}}$ as calculated with formula $\mathrm{n} .1$ for the absorption spectra in figure $\mathrm{c}$.

We define a leading parameters for the study of the CD in this sample in order to get a valuable reference:

$$
C D_{A} \lambda=2 \frac{A_{R} \lambda-A_{L} \lambda}{A_{R} \lambda+A_{L} \lambda}
$$

The $\mathrm{CD}_{\mathrm{A}}$ is evaluated both in case of upward or forward illumination. After few calculations, we know that the two version of the same parameter are related by:

$$
C D_{A}^{\downarrow} \lambda \approx C D_{A}^{\uparrow} \lambda \frac{1-\frac{1-R_{H} R_{S}}{1-R_{S}^{2}}}{\left[1+\frac{1-R_{H} R_{S}}{1-R_{S}^{2}}\right]-\frac{2 R_{S}}{1-R_{S}^{2}} \frac{A_{H, R} \cdot A_{H, L}}{A_{H, R}+A_{H, L}}}
$$

where $R_{H}$ and $R_{S}$ refer to the reflectivity of the helices' layer and substrate, respectively, and the $A_{H, R / L}$ refer to the absorbance of the helices' layer without the substrate. With the calculated values with our simulations, the second factor at the right hand side of (2) is approximately one, thus the $\mathrm{CD}_{\mathrm{A}}$ as calculated by illuminating the samples either from above the helices or from below the substrate must present almost the same values, as reported also by the plot in fig. $4 \mathrm{~d}$. In the same figure, three dotted vertical lines indicate 3 corresponding wavelengths $(633 \mathrm{~nm}, 808 \mathrm{~nm}, 980 \mathrm{~nm})$ where we measured the PA signals. Being the crossing point approximately located at $890 \mathrm{~nm}$ in fig. $4 \mathrm{~d}$, we expect similar results for the first two wavelengths and an opposite effect for the third one.

For the sake of deeper investigation, we also extracted the 3D maps for the ohmic losses, defined as:

$$
P_{\Omega} \lambda=\frac{1}{2} \operatorname{Re} \vec{J} \lambda \cdot \vec{E} \lambda^{*}
$$

where $J$ is the electric volume current density, $E$ is the electric field, and * denotes the operation of conjugation. The 3 corresponding distributions are plotted in fig.5:
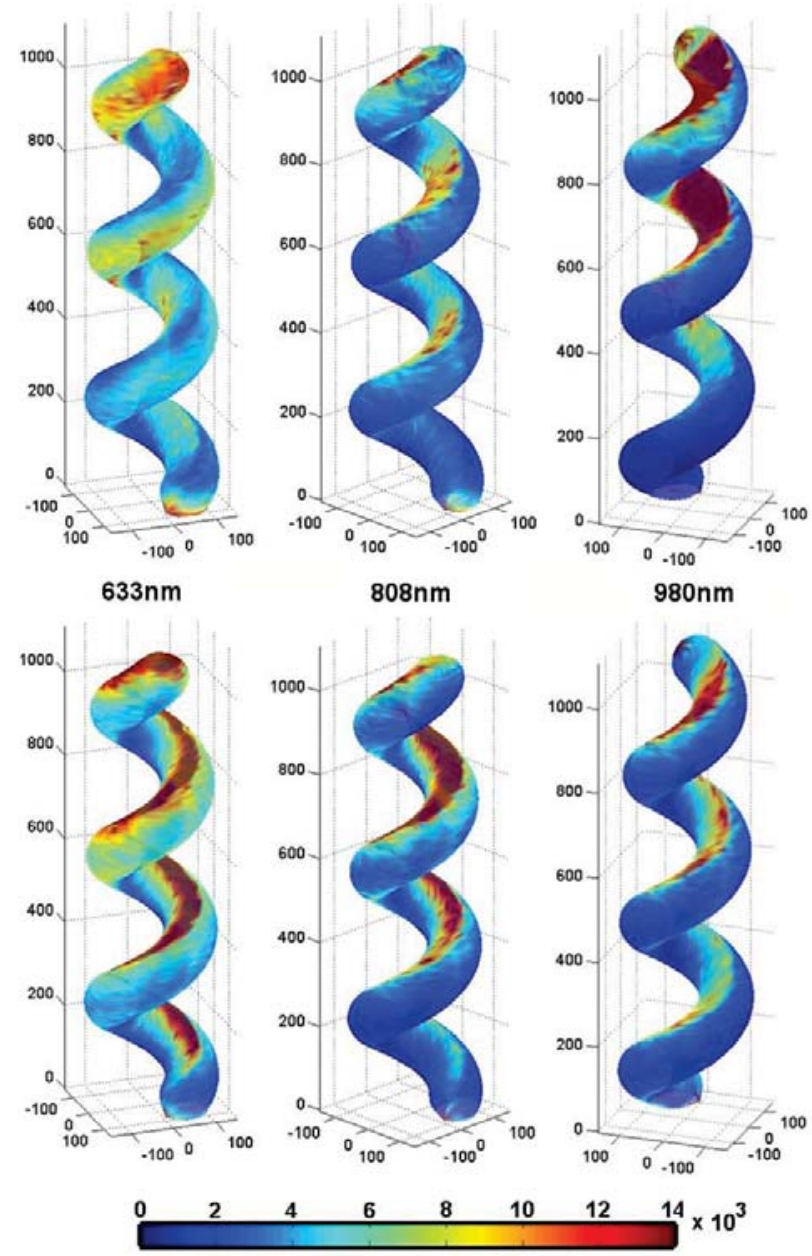

Figure 5. Surface plots of the ohmic losses as defined in formula n.3. The geometric scale is in $\mathrm{nm}$, and the colorbar in $\mathrm{W} / \mathrm{m}^{3}$. In the first (second) row we plotted the spatial distributions for left (right) circular polarization. To avoid problems coming from the field discontinuites across the surface, the field values have been retrieved $3 \mathrm{~nm}$ below the surface level. It is clear how strong is the ohmic dissipation of power for the right polarization at $\lambda=633 \mathrm{~nm}$ and $808 \mathrm{~nm}$ and for the left polarization at $\lambda=980 \mathrm{~nm}$. In this last case, the dissipation is mostly located at the first of the helix. All this maps have been calculated under downward illumination.

\section{Experimental data and final comparisons.}

The PA measurements essentially confirmed the results of the all-optical measurements published in ref.[], and the calculated values for the $\mathrm{CD}_{\mathrm{A}}$ are close to the ones calculated by numerical methods. In fig. 6 we plot the main data for the wavelengths above mentioned, obtained by continuously rotating the quarter phase plate (QPP). Values of the QPP orientation equal to $45^{\circ}$ (plus an integer multiple of $180^{\circ}$ correspond to the left circular polarization, while QPP values of $135^{\circ}$ (and multiples of $180^{\circ}$ ) correspond to the right circular polarization. Our definitions for the right and left circular senses follow the ones of the standard IEEE [9]. 

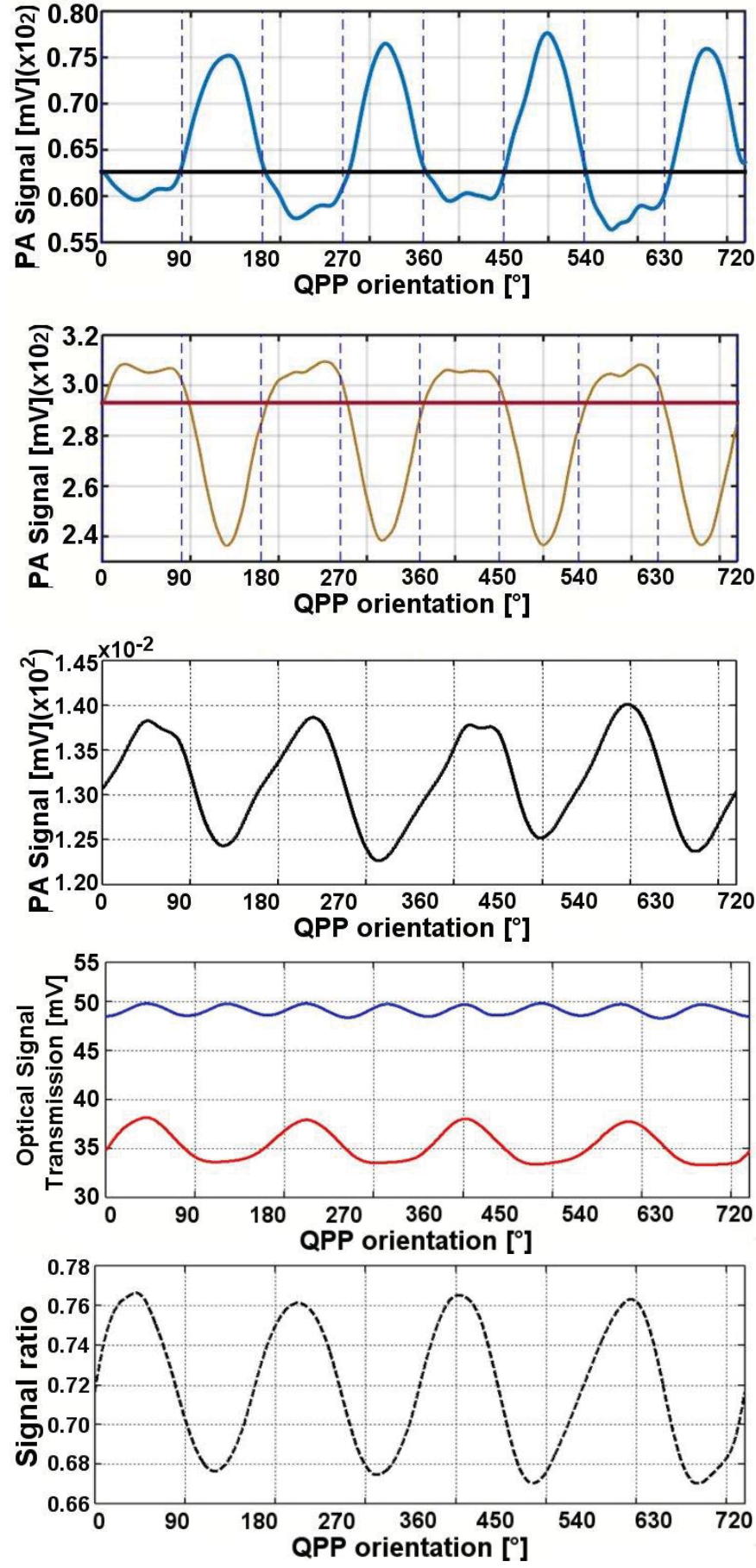

Figure 6. (a) PA signal at $\lambda=633 \mathrm{~nm}$, (b) PA signal at $\lambda=980 \mathrm{~nm}$ for downward illumination, (c) PA signal at $\lambda=980 \mathrm{~nm}$ for upward illumination, (d) optical transmission signal $(\mathrm{T})$ at $\lambda=633 \mathrm{~nm}$ (blue for $\mathrm{T}$ evaluated in a region occupied only by the substrate, red for a region prevalently occupied by the sample), and (e) signal ratio calculated from the twe previous signals. The PA data for $\lambda=808 \mathrm{~nm}$ are quite the same for the case of $\lambda=633 \mathrm{~nm}$. Note that the upper 3 plots should be perfectly sinusoidal in shape, but they are not so because of the intrinsic linear anisotropy of the substrate, as reported in the blue profile in (d). Indeed, the structure-to-pure substrate transmission ratio in (e) presents a perfect sinusoidal profile. Calculated $\mathrm{CD}_{\mathrm{A}}$ values for the 3 cited wavelengths after the exclusion of the substrate's anisotropy are 0.36 , 0.34 and -0.25 , close to their numerical counterpart, which are $0.5,0.33$ and -0.25 . This latter value changes consistently in case of upward illumination, reducing its value to -0.12 , as can be seen by a further inspection of the black plot in (c).
After a brief inspection, we firstly note that the PA signal plots perfectly respects the major absorption ratios of the right circular polarization both for $\lambda=633 \mathrm{~nm}$ and $\lambda=808 \mathrm{~nm}$. At $\lambda=980 \mathrm{~nm}$ the situation is reversed, in accordance with the plot of fig. 4 d. Despite that, we note also that the PA signal profile is not perfectly sinusoidal in shape as expected. This fact is due to the substrate's linear anisotropy, as shown in fig.6d. By ideally deleting the effects of the anisotropy, the extrapolated values of the $\mathrm{CD}_{\mathrm{A}}$ are quite the same as calculated by means of the numerical simulations. We also tested the ideal independence of the $\mathrm{CD}_{\mathrm{A}}$ from the direction of illumination. Anyway, the measured PA signal for upward illumination, other than being naturally weaker than that for the opposite case, reported a minor $\mathrm{CD}_{\mathrm{A}}$ norm of 0.12 . We can explain this minor value with the thermal conductivity effect due to the upper substrate layer, which partially dissipates the heat produced at the helix tip facing the input field and connected with the substrate, as can be easily expected by vertically reversing the 3D maps of fig.5.

\section{Conclusions.}

The PA measurements confirmed the results of all-optical measurements and numerical simulations. Also secondary effects related to heat diffusion in the substrate are in perfect agreement with the 3D simulated profiles of the ohmic losses, which explain why the heat transmission to the surrounding air is less efficient and consequently the recorded sound signal appears to be weaker. The PA technique emphasizes the e.m. field-helices interaction which is the only one associated with the sound generation, thus avoiding all the problems coming from the averaging the transmitted field with the one escaping from regions featuring only pure substrate and no helices, as happens for all-optical techniques.

\section{References}

[1] J. K. Gansel, M. Thiel, M. S. Rill, M. Decker, K. Bade, V. Saile, G. von Freymann, S. Linden, M. Wegener, "Gold Helix Photonic Metamaterial as Broadband Circular Polarizer", Science, Vol.325, No.5947, pp.1513-1515 (2009).

[2] M. Esposito, V. Tasco, F. Todisco, A. Benedetti, D. Sanvitto, A. Passaseo, "Three Dimensional Chiral Metamaterial Nanospirals in the Visible Range by Vertically Compensated Focused Ion Beam InducedDeposition", Adv. Opt. Mat., Vol.2, Iss.2, pp.154-161(2014).

[3] M. Esposito, V. Tasco, M. Cuscunà, F. Todisco, A. Benedetti, I. Tarantini, M. De Giorgi, D. Sanvitto, A. Passaseo, "Nanoscale 3D Chiral Plasmonic Helices with Circular Dichroism at Visible Frequencies", ACS Photonics, Vol.2, No.1, pages 105-114 (2014).

[4] M. Esposito, V. Tasco, F. Todisco, M. Cuscunà, A. Benedetti, D. Sanvitto, A. Passaseo, " Triple-helical nanowires by tomographic rotatory growth for chiral photonics", Nature Comm., Vol.6, No.6484 (2015).

[5] R. Inagaki, K. Kagami, E.T. Arakawa, "Photoacoustic study of surface plasmons in metals", Appl. Opt., Vol. 21, Issue 5, pages 949-954 (1982).

[6] A. Miklós, S. Schäfer, P. Hess, "Photoacoustic Spectroscopy, Theory", pp.1815-1822, University of Heidelberg, Germany, Academic Press (1999).

[7] S. K. Kulkarni, "Nanotechnology: Principles and Practices", Springer (2014).

[8] http://www.bksv.com/doc/Bp0100.pdf.

[9] IEEE Std 149-1979 (R2008), "IEEE Standard Test Procedures for Antennas". Reaffirmed December 10, 2008, Approved December 15, 1977, IEEE-SA Standards Board. Approved October 9, 2003, American National Standards Institute. ISBN 0-471-08032-2. doi: 10.1109/IEEESTD.1979.120310. 